\title{
Characteristics of changes and clinical and instrumental predictors of the severity of structural remodelling of carotid arteries in hypertensive patients
}

\author{
Nataliia V. Kuzminova', Nataliia Y. Osovska', Sergiy E. Lozinsky', Iryna I. Knyazkova ${ }^{3}$ \\ 'Department of Internal Medicine Nr.1, Vinnitsa National Medial University named after M. Pyrogov, Vinnitsa, Ukraine \\ ${ }^{2}$ Department of Internal Medicine of the Medical Faculty Nr. 2, Vinnitsa National Medial University named after M. Pyrogov, Vinnitsa, Ukraine \\ ${ }^{3}$ Department of Clinical Pharmacology, Kharkiv National Medical University, Kharkiv, Ukraine
}

\begin{abstract}
Background. Mechanisms of activation of the process of vascular wall remodelling in patients affected by arterial hypertension have not been studied in depth and require clarification.

Materials and methods. The study included 381 patients with hypertension -212 men and 169 women of the average age $53.0(47 ; 60)$ years. The structural-functional vessel status was determined by the method of duplex scanning and colour duplex mapping of blood flow with the Logiq $500 \mathrm{MO}$ apparatus (GE, USA). Statistical analyses were made using Microsoft Excel software kit, Statistica for Windows 6.0.

Results. The patients with hypertension presented some left-right asymmetry of remodelling extracranial carotid arteries. Unlike the impact of remodelling of the right carotid artery, the most essential effect on the left carotid artery was the impact of daytime pulse arterial pressure and variability of the nocturnal systolic arterial pressure (the strength of impact 25.0 and $13.9 \%$, respectively. The processes of remodelling of the right carotid artery are more sensible to the impact of high values of nocturnal diastolic arterial pressure (the strength of impact 16.4\%). The beginning of some brain complication is associated with the significant increase in atherosclerotic affection not only of the left, but also of the right carotid artery.

Conclusions. Remarkable remodelling of the right carotid artery is often associated with the severity of the disease and to some extent reflects the severity of the flow of the disease and can be regarded as an additional unfavourable feature.

key words: hypertonic disease, carotid arteries, vessel remodelling, atherosclerosis, predictors Arterial Hypertens. 2016, vol. 20, no. 2, pages: 60-67 DOI: $10.5603 / A H .2016 .0010$
\end{abstract}

\section{Introduction}

One of the reasons for the increased peripheral resistance in arterial hypertension $(\mathrm{AH})$ is the change in the properties of the vascular wall, the so-called vascular remodelling. It is an important mechanism that is responsible for the process of improvement of vascular reserve, autoregulation of cerebral blood flow and the development of atherosclerosis [1-6]. The vascular lesions in hypertension involve endothelial dysfunction, thickening of the large arteries intima-media, especially the carotid. This results in

Address for correspondence: Nataliia V. Kuzminova, MD 42, app. 91, Kosmonavtiv Street, Vinnitsa, 21027, Ukraine Tel.: (+380432) 52-22-37, tel. mobile: (+38 050) 442-47-07 e-mail: kuzminova5507@mail.ru

V M Copyright (C) 2016 Via Medica, ISSN 2449-6170 
the development and progression of atherosclerosis $[2,5,7]$. The walls of the arteries thicken and become rigid. The lumen of the vessel is changed. Often the arteries elongate and become convoluted. A decrease in organ perfusion, including the brain, is observed $[1,2,7,8]$. The thickness of the intima-media complex in carotid arteries (CA) is considered one of the independent risk factors of transient ischaemic attacks and stroke. The relationship between type of ischaemic stroke, its localization, extent of the ischaemic damage foci and the intima-media thickness (IMT) were demonstrated $[9,10]$. However, it is believed that the importance of IMT as a risk factor of vascular events is not completely defined [11]. Kazmierski et al. (2004) confirmed the role of compensatory thickening of IMT to ensure the adequacy of blood flow [12].

The activation mechanisms of the remodelling process in the vascular wall are not reliably found. It is known that vascular remodelling in hypertension is not necessarily accompanied by a significant increase in the number of cells or mass of the medial layer. Changes of the blood vessel lumens can occur as a result of a combination of cell proliferation and apoptosis, activation of the synthesis of connective tissue matrix and/or its degradation [1]. The processes of hypertrophy and proliferation of smooth muscle cells of the blood vessels can be influenced by adrenergic stimulation, platelet growth factor, changes of the matrix metalloproteinase activity, urokinase plasminogen activator and many other factors $[1,7]$. Consequently, in the genesis of cerebrovascular and other cardiovascular $(\mathrm{CV})$ complications in essential hypertension $(\mathrm{EH})$ the role of remodelling of blood vessels, which supply blood to the brain, and the activation mechanisms of the remodelling process in the vascular wall in hypertensive patients are not fully understood and require further definition and clarification.

The purpose of the study was to reveal the peculiarities of structural changes of the vascular wall and independent clinical and instrumental predictors of the severity of extracranial carotid arteries structural remodelling in hypertensive patients.

\section{Materials and methods}

Overall, 381 patients with EH were examined. There were 212 males and 169 females, aged from 35 to 70 years, the mean age was $53.0(47 ; 60)$. Stage II hypertension was diagnosed in $231(60.6 \%)$ patients, stage III — in 150 (39.4\%) patients. There was a combination of hypertension with coronary heart disease (CHD) in 259 (68.0\%) patients: stable angina of the II functional class (FC) was found in $56(14.7 \%)$ subjects, stable angina of FC III - in 196 (51.4\%). Eighty-eight (23.1\%) patients had previous history of myocardial infarction (MI). Forty-five $(11.8 \%)$ patients suffered from acute cerebrovascular accident (ACVA). The combination of MI and stroke was found in 17 (4.5\%) patients. The vast majority of patients had $\mathrm{AH}$ of the 1st, 2nd and 3rd degree: 16 (4.2\%); 292 (76.6\%) and 73 (19.2\%) patients, respectively. Chronic heart failure (CHF) of FC I-II [according to the criteria of the New York Heart Association (NYHA)] was diagnosed in 175 (45.9\%) patients, III FC - in 163 (42.8\%) patients. Duration of hypertension averaged $10.1 \pm$ 0.32 years. Normal geometry was established in 66 patients $(17.4 \%)$, concentric remodelling - in 90 (23.6\%), concentric LVH - in $156(40.9 \%)$ and eccentric $\mathrm{LVH}$ - in 69 (18.1\%) patients. Diagnosis of $\mathrm{EH}$ and $\mathrm{CHD}$ was established according to the recommendations of the European Society of Hypertension and European Society of Cardiology ESH/ /ESC (2007) [13]. The study did not include patients with history of MI or stroke less than 3 months prior to the study, subjects with liver or kidney dysfunction, diabetes, heart disease, chronic heart failure of the FC IV, chronic obstructive pulmonary disease and respiratory failure. The control group included 30 healthy subjects of similar age and gender.

Prior to the research, screening, thorough acquisition of complaints and anamnesis had been performed as well as patients' agreement had been obtained to participate in the research according to the ethic norms of the Helsinki Declaration.

Evaluation of structural and functional state of the heart was performed based on echocardiography data (Echo) [14-17]. Daily blood pressure profile was assessed using ambulatory blood pressure monitoring (ABPM) [18]. Structural and functional state of vessels was assessed by duplex scanning and colour Doppler mapping of blood flow [19, 20]. To study the functional state of the endothelium of blood vessels, all patients underwent Doppler ultrasound of the brachial artery [21].

Statistical calculations were performed using the software Microsoft Excel and "Statistica" for Windows 6.0. Average values were expressed as Me (II), where $\mathrm{Me}$ - median, II - interquartile interval. Determination of reliability of differences between groups was performed using Mann-Whitney test. A comparative analysis of differences was performed according to Pearson criterion $\chi^{2}$ [22]. Aggregate indicators of the severity of structural remodelling of the CA were calculated to determine the inde- 
pendent predictors of structural remodelling of the left and right CA. Overall, 381 clinical cases of EH of the II-III stages and 132 different clinical and instrumental parameters were used to form basic statistical matrix. Two summary indices (defined as the sum of scores) were analysed as the output parameter: 1) severity of the right CA structural remodelling (SRrca) and 2) severity of the left CA structural remodelling (SRlca). Table I shows the method of summary results calculation. For indicators of SRlca and SRrca, the minimum total score was 0 points and the maximum -7 points. The average values of SRrca and SRlca were $3.2 \pm 0.02$ and $3.4 \pm 0.03$, respectively. Multiple step-by-step regression (module "Multiple Regression" of the package StatSoft "Statistica" V. 5.5) was used to conduct multivariate analysis [22].

Multivariate analysis was presented as the form of linear regression models of type $\mathrm{Y}=\mathrm{f}(\mathrm{X} 1, \mathrm{X} 2$, ..., Xn), where $\mathrm{X} 1, \mathrm{X} 2, \ldots, \mathrm{Xn}$ were the independent variables that characterized clinical and functional state of patients and $\mathrm{Y}$ was the output parameter of the severity of carotid arteries structural remodelling. Taking into account the large number of indicators for analysis, we have developed two types of models - clinical and instrumental, that influenced the severity of structural remodelling of the left and right carotid arteries. The clinical and instrumental parameters were considered as independent predictors, if they showed significant $(\mathrm{p}<0.05)$ association with the source parameter. The informativeness of complex influence of factors on the output parameter was estimated by multiple regression coefficient calculation (coefficient of determination $-\mathrm{R}$ ); the adequacy — utilizing Residual analysis for calculation of actual and critical (df) value of Fisher test (F-test, and significance level (p); specificity - using the error calculation of the impact factors (St. error of estimate). The following tables contain obtained models with the minimum and maximum informativeness. Beta-coefficient ( $\beta$-coefficient) was used for statistical characteristics of the independent predictors that showed the strength of factor influence on the output parameter and the nature of that impact (a positive $\beta$-coefficient testified for the direct correlation and a negative $\beta$-coefficient - for the feedback). In addition to the $\beta$-coefficient, the strength of influence of selected indicators on the degree of impairment of the structural and functional state of the myocardium was determined. For greater clarity, the influence of individual factors on the output parameter was represented as a percentage (counted as the ratio of the $\beta$-factor to the sum of all $\beta$-coefficients) [22].
Table I. The method of summary results calculation

\begin{tabular}{|l|c|}
\hline Criteria & Scores \\
\hline $\begin{array}{l}\text { The total index of structural remodelling severity of the left and } \\
\text { right carotid arteries (SRIca and SRrca) }\end{array}$ \\
\hline \multicolumn{2}{|l|}{ The presence of atherosclerotic plaques } \\
\hline \multicolumn{2}{|l|}{ Plaque absent } \\
\hline Plaque(s) present & 1 \\
\hline \multicolumn{2}{|l|}{ The presence and nature of carotid artery stenosis } \\
\hline Stenosis absent & 0 \\
\hline Stenosis $<50 \%$ of vessel lumen & 1 \\
\hline Stenosis $>50 \%$ of vessel lumen & 2 \\
\hline The presence of deformations of the carotid arteries \\
\hline Deformation not observed & 0 \\
\hline Deformation(s) of carotid arteries present & 1 \\
\hline The value of intima-media thickness (IMT) & \\
\hline IMT $\leq 0.6 \mathrm{~mm}$ & 0 \\
\hline IMT $=0.7-0.9$ mm & 2 \\
\hline IMT $>0.9 \mathrm{~mm}$ & 3 \\
\hline
\end{tabular}

\section{Results and discussion}

During the analysis of structural and functional extracranial arteries it was determined that the prevalence of CA atherosclerotic lesions significantly increased in patients with stage II hypertension without coronary artery disease (47.3\%) compared with the control group (8.0\%). The fact of the asymmetric defeat of CA was discovered. According to the literature review this fact has not received an adequate attention. So, according to our results, a more distinct change of the group with $\mathrm{EH}$ without IHD was observed in the left CA: the features of atherosclerotic lesions of the left and right CA were identified in $35(31.8 \%)$ and $17(15.5 \%)$ patients, respectively, $\mathrm{p}<0.05$ [23]. A clear tendency to increase the frequency of defeat of right $\mathrm{CA}$ in combination of $\mathrm{EH}$ and IHD was observed, compared with EH patients without IHD $(p=0.08)$. Marked thickening of the intima-media (IMT) was observed both in the II and III stage of EH. More significant changes of IMT were found in patients with EH of the III stage. Also, the absolute value of IMT progressively increased with age and duration of hypertension.

The degree of atherosclerotic lesions of the left CA significantly increased from $24.9 \%$ to $41.9 \%$ ( $\mathrm{p}<$ 0.05 ) with the increase of the degree of hypertension (from the 2nd to $3 \mathrm{rd}$ ) in patients with stage II hypertension. The defeat of the right $\mathrm{CA}$ remained roughly at the same level as at the II degree of $\mathrm{AH}$. This 
indicates that the increase of BP leads to preferential damage of the left $\mathrm{CA}$, which is experiencing greater haemodynamic influence due to anatomical features - as it originates directly from the aortic arch. The increase in the number of atherosclerotic plaques with higher values of BP in patients with stage II hypertension was accompanied by an increase in the prevalence of the left CA stenosis ( $>30 \%$ of the lumen), $\mathrm{p}<0.05$. The prevalence of the right CA stenosis in this group of patients did not meet the confidence level in relation to patients with stage II hypertension and moderate hypertension [24]. It should be noted that in patients with stage II hypertension and severe hypertension the number of deformations of the right CA [in the form of the curves (angular and accompanying) and gyri] was significantly increased $(p=0.009)$. The increasing prevalence of similar deformities of the left CA during the III degree of $\mathrm{AH}$ was on the verge of confidence $(p=0.06)$. Marked thickening of both the left and right CA was found, but without significant differences between groups of patients with different degrees of hypertension. Diameters of both CA were significantly increased in patients with uncomplicated $\mathrm{EH}$, but with severe hypertension, compared with the group of patients with moderate hypertension $(p<0.05)$. A decrease of linear blood flow velocity (LBFV) at the 3rd degree of $\mathrm{AH}$ in the left CA was observed $(\mathrm{p}<0.05)$. Thus, remodelling of extracranial arteries occurs primarily with the growth of the degree of hypertension in patients with stage II hypertension due to left CA, which is experiencing greater haemodynamic load. Changes in the right CA have the same direction, but do not reach the validity in relation to the group of patients with moderate hypertension. Remodelling of the right CA is mainly due to the increase of the number of different strains.

Further progression of the disease, the appearance of $\mathrm{EH}$ complications were accompanied by the progression of the structural and functional restructuring of the CA wall. So, there was a significant increase in the rate of atherosclerotic plaques in extracranial arteries of patients with EH of the III stage and moderate hypertension compared with the group of patients with EH of the II stage and a similar degree of hypertension. It should be noted that a significant increase in the number of plaques was observed not only in the left, but also in the right CA: $50.5 \%$ versus $24.9 \%(\mathrm{p}<0.0001)$ in the left $\mathrm{CA}$ and left $34.0 \%$ versus $20.1 \%(\mathrm{p}=0.009)$ in the right $\mathrm{CA}$, respectively $[4,23]$. The revealed changes confirm the influence on the process of atherosclerotic vascular damage not only the magnitude of blood pressure, but also other factors, which increase the severity of the disease. Significant progression of the atherosclerotic process in the right $\mathrm{CA}$ in patients with $\mathrm{EH}$ of the III stage and moderate hypertension has led to a substantial reduction of LBFV [to 0.76 $(0.66-0.84) \mathrm{m} / \mathrm{s}$ ] compared with the group with stage II hypertension and similar levels of blood pressure.

The frequency of CA atherosclerotic lesions was significantly increased in patients with $\mathrm{EH}$ of the stage III compared with EH of the stage II. More expressive changes were determined in the left CA throughout the group of patients with $\mathrm{EH}$ of the stage III: $82(54.7 \%)$ versus $48(32.0 \%)$ in the right CA ( $p<0.0001)$. The frequency of stenosis and deformation of CA significantly increased in patients with $\mathrm{EH}$ of the stage III. When evaluating the frequency of atherosclerotic lesions of CA, depending on the nature of the complications, the predominant lesion by the atherosclerotic process was observed in the left CA compared with right one in patients without cerebral catastrophes in history: 15 (44.1\%) in the left CA versus 7 (20.6\%) in the right CA in the group of patients without myocardial infarction (MI) and CHF IIA, respectively ( $\mathrm{p}<0.05)$, and 38 $(53.5 \%)$ versus $17(23.9 \%)$, respectively, in patients with cardiosclerosis after MI ( $<$ < 0.0003) [25]. In the analysis of structural and functional parameters of $\mathrm{CA}$ that were obtained from the patients with $\mathrm{EH}$ of the stage III, who had history of acute cerebrovascular disease (ACVD), it was determined that in patients with cerebral stroke (MI) there was a gradual increase in the frequency of atherosclerotic injury of the right CA compared with patients who did not have history of brain accidents: $42.9 \%$ in the group with ACVD compared with $23.9 \%$ in patients with IM ( $\mathrm{p}<0.05)$, and $20.6 \%$ in patients without MI and MI $(\mathrm{p}<0.05)$, respectively. Prevalence of the right CA damage in patients with a history of a brain catastrophe significantly increased and almost equalled to the prevalence of the left CA lesions. Significant difference between the number of atherosclerotic plaques in the left and right CA was not determined in patients with a history of cerebral stroke [ $50.0 \%$ versus $42.9 \%$, respectively ( $p=0.59)$ ] and in patients with the history of MI and stroke $[88.2 \%$ vs $70.6 \%$, respectively $(p=1.62)]$. So, the atherosclerotic lesions were almost identical in the left and right CA. The increase in the number of atherosclerotic plaques, mostly in the right $\mathrm{CA}$, was accompanied by the process of stenosis, that led to a significant reduction of the lumen of the vessel and has gained confidence in comparison with MI group $(\mathrm{p}=0.011)$ and group without $\mathrm{MI}$ and stroke $(\mathrm{p}=$ $0.013)$, respectively. In parallel with the progression 
of the atherosclerotic process and the development of haemodynamically significant stenosis, statistically significant increase in the number of different strains in the right CA occurred in patients with ACVD compared with patients with EH who had not had brain complications. The highest prevalences of atherosclerotic plaques in the right $\mathrm{CA}$ in parallel with the increase of atherosclerotic plaques in the left CA were registered in patients with a double disaster: MI and stroke. Thus, the appearance of cerebral complications was associated with a significant increase of atherosclerotic lesions not only in the left, but also in the right CA. Significant remodelling of the right CA was often associated with the severity of the disease. Moderate and direct correlation between the presence of plaques in right CA and systolic dysfunction of the left ventricle was identified $(r=0.27$, $\mathrm{p}=0.002)$. Also, it was a correlation between stenosis of the right $\mathrm{CA}$ and systolic dysfunction of the left ventricle $(\mathrm{r}=0.23, \mathrm{p}=0.01)$ [25].

Thus, increase of the right CA remodelling reflects the severity of the disease and can be regarded as an additional adverse prognostic sign. So, in the study of the extracranial vessels remodelling process and factors associated with it, we revealed noticeable differences in the degree of changes and in the time of their occurrence in the left and right common CA. It can be assumed that this is due to different influence of clinical and instrumental parameters on the structural remodelling of extracranial vessels, which was confirmed by the results of a multivariate regression analysis.

On the right CA remodelling the most pronounced influence provided such clinical factors as patient's age, stage of hypertension and waist circumference (impact force is equal to $55.7 \% ; 30.3 \%$; $14.0 \%$, respectively). The explanations are given in Tables II and III.

From the parameters identified by instrumental methods, the greatest influence was exerted by the presence of diastolic and systolic LV dysfunction

Table II. Clinical models of the severity of the right CA structural remodelling in patients with $\mathrm{EH}$

\begin{tabular}{|l|}
\hline Step 1 \\
SRrca $=-2.19+0.10$ Age \\
$R=0.62, p<0.0001 ;$ St. error of estimate $=1.97$ \\
\hline Step 2 \\
SRrca $=-4.57+0.10$ Age +0.95 EHst \\
$R=0.73, p<0.0001 ;$ St. error of estimate $=0.85$ \\
\hline Step 3 \\
SRrca $=-7.00+0.11$ Age +0.90 EHst $+0.023 \mathrm{WC}$ \\
$R=0.75, p<0.0001 ;$ St. error of estimate $=0.83$ \\
\hline
\end{tabular}

Notes: 1. Age - patient's age in years; 2 . EHst — binary measure coded in points, characterizing clinical stage of $\mathrm{EH}$ (2 points mean $\mathrm{EH}$ of the stage II, 3 points - EH of the stage III); 3 . WC - waist circumference in $\mathrm{cm}$
(DT and EF, respectively, as its value); impairment of vasodilative vascular endothelial function evidenced by a reduced response of the brachial artery to decompression test, as well as a small degree of night decrease of NDP in \%. The power of influence of these factors was equal to $29.8 \% ; 31.7 \% ; 22.1 \%$; $16.4 \%$, respectively (Tables IV and V).

Analysis of the impact of studied clinical parameters on remodelling of the left CA showed common factors with the right carotid artery. They are the following: patient's age, waist circumference, stage of $\mathrm{EH}$ (the strength of the influence was equal to $48.0 \%$; $20.3 \% ; 18.7 \%$, respectively). However, the strength of the influence of atrial fibrillation significantly increased (13.0\%), which was not observed in the analysis of the remodelling process of the right CA (Tables VI and VII).

Usually atrial fibrillation (AF) occurs in patients with severe $\mathrm{LVH}$, a long course of $\mathrm{EH}$ that may be accompanied by changes in the myocardium and vascular wall. This is the evidence of the presence of correlation between LVH and IMT in patients with severe EH. It was noted that remodelling of the left CA occurred earlier and was more pronounced than of the right one. This may be explained by more pronounced haemodynamic stress due to anatomical features of left $\mathrm{CA}-$ it originates from the aortic

Table III. The analysis of clinical predictors of the severity of the right CA structural remodelling

\begin{tabular}{|l|c|c|c|}
\hline The factor & $\beta$-coefficient & $\mathbf{R}$ & $\begin{array}{c}\text { Strength of the } \\
\text { influence (\%) }\end{array}$ \\
\hline Age & 0.68 & 0.000003 & 55.7 \\
\hline EHst & 0.37 & 0.0001 & 30.3 \\
\hline WC & 0.17 & 0.008 & 14.0 \\
\hline
\end{tabular}

Table IV. Instrumental models of the severity of the right CA structural remodelling in patients with $\mathrm{EH}$

\begin{tabular}{|c|}
\hline $\begin{array}{l}\text { Step } 1 \\
\text { SRrca }=1.04+0.013 \text { DT } \\
R=0.40, p=0.013 ; \text { St. error of estimate }=1.15\end{array}$ \\
\hline $\begin{array}{l}\text { Step } 2 \\
\text { SRrca }=3.95+0.014 \mathrm{DT}-0.05 \mathrm{EF} \\
\mathrm{R}=0.53, \mathrm{p}=0.003 ; \text { St. error of estimate }=1.08\end{array}$ \\
\hline $\begin{array}{l}\text { Step } 3 \\
\text { SRrca }=3.78+0.012 \text { DT }-0.05 \text { EF }+0.62 \text { RVBAd } \\
R=0.58, p=0.002 ; \text { St. error of estimate }=1.04\end{array}$ \\
\hline $\begin{array}{l}\text { Step } 4 \\
\text { SRrca }=4.28+0.011 \text { DT }-0.05 \text { EF }+0.57 \text { RVBAd }-0.02 \text { DI DBP } \\
R=0.61, p=0.002 ; \text { St. error of estimate }=1.01\end{array}$ \\
\hline
\end{tabular}


Table V. Analysis of instrumental predictors of the right CA structural remodelling severity in patients with $\mathrm{EH}$

\begin{tabular}{|l|c|c|c|}
\hline The factor & $\beta$-coefficient & $\mathbf{R}$ & $\begin{array}{c}\text { Strength of the } \\
\text { factor influence (\%) }\end{array}$ \\
\hline DT & 0.31 & 0.003 & 29.8 \\
\hline EF & -0.33 & 0.002 & 31.7 \\
\hline RVBAd & 0.23 & 0.011 & 22.1 \\
\hline DI DBP & -0.17 & 0.023 & 16.4 \\
\hline
\end{tabular}

Table VI. Clinical models of the severity of left CA structural remodelling in patients with $\mathrm{EH}$

\begin{tabular}{|l|}
\hline Step 1 \\
SRIca $=-1.69+0.093$ Age \\
$\mathrm{R}=0.54, \mathrm{p}<0.0001 ;$ St. error of estimate $=1.10$ \\
\hline Step 2 \\
SRrca $=-6.72+0.11$ Age $+0.05 \mathrm{WC}$ \\
$\mathrm{R}=0.61, \mathrm{p}<0.0001 ;$ St. error of estimate $=1.04$ \\
\hline Step 3 \\
SRrca $=-7.68+0.11$ Age $+0.04 \mathrm{WC}+0.60 \mathrm{EH}$ st \\
$\mathrm{R}=0.65, \mathrm{p}<0.0001 ;$ St. error of estimate $=1.01$ \\
\hline Step 4 \\
$\mathrm{SRrca}=-7.00+0.10$ Age $+0.037 \mathrm{WC}+0.60$ EHst +0.73 \\
AF \\
$\mathrm{R}=0.69, \mathrm{p}<0.0001 ;$ St. error of estimate $=0.98$ \\
\hline Notes: 1 . Age - patient's age in years; 2 . WC - waist circumference in $\mathrm{cm} ; 3$. EHst - stage of EH \\
in points (2 and 3 points mean the stage II of EH); 4 . AF - a binary feature which characterizes the \\
presence of AF episodes (0 points - no history of AF; 1 point - prior history of AF episodes)
\end{tabular}

arch. However, the progression of the disease significantly activated remodelling of the right CA.

Differences in instrumental parameters which influence the processes of the left CA remodelling were more pronounced compared with those of the right one (Tables VIII and IX). Such factors as the decline of EF, impairment of the vasoactive function of endothelium (reduction of increase of the brachial artery diameter in the test with decompression), diastolic dysfunction of the left ventricle (the force of the impact was equal to $31.5 \% ; 14.8 \%$ and $14.8 \%$, respectively) continued to be important for remodelling of the left and the right CA. In contrast to the right $\mathrm{CA}$ remodelling the daily $\mathrm{PBP}$ and variability of the night CBP was significant for the left CA (the power of influence was equal to $25.0 \%$ and $13.9 \%$, respectively). It was supposed that increasing values of $\mathrm{BP}$ were more conductive to remodelling of the left CA than of the right one.

Recent studies have convincingly demonstrated the adverse role of peripheral PBP in the development of $\mathrm{CHD}, \mathrm{CHF}$, chronic renal failure and increased cardiovascular risk. It allowed the experts of ESH to attribute PBP to the risk factors of adverse prognosis in patients with hypertension in older age
Table VII. The analysis of clinical predictors of the severity of the left CA structural remodelling in patients with $\mathrm{EH}$

\begin{tabular}{|l|c|c|c|}
\hline The factor & $\beta$-coefficient & $\mathbf{R}$ & $\begin{array}{c}\text { Strength of the } \\
\text { factor influence (\%) }\end{array}$ \\
\hline Age & 0.59 & 0.000001 & 48.0 \\
\hline WC & 0.25 & 0.003 & 20.3 \\
\hline EHst & 0.23 & 0.003 & 18.7 \\
\hline AF & 0.16 & 0.014 & 13.0 \\
\hline
\end{tabular}

Table VIII. Instrumental models of the severity of left CA structural remodelling in patients with $\mathrm{EH}$

\begin{tabular}{|c|}
\hline $\begin{array}{l}\text { Step } 1 \\
\text { SRIca }=7.11-0.065 \mathrm{EF} \\
\mathrm{R}=0.39, \mathrm{p}=0.014 ; \text { St. error of estimate }=1.24\end{array}$ \\
\hline $\begin{array}{l}\text { Step } 2 \\
\text { SRIca }=7.97-0.075 \mathrm{EF}-0.04 \% \Delta d \text { br. art. } \\
R=0.50, p=0.006 ; \text { St. error of estimate }=1.19\end{array}$ \\
\hline $\begin{array}{l}\text { Step } 3 \\
\text { SRlca }=5.63-0.086 \mathrm{EF}-0.039 \% \Delta d \text { br. art. }+0.047 \\
\text { DPAP } \\
R=0.57, p=0.003 ; \text { St. error of estimate }=1.14\end{array}$ \\
\hline $\begin{array}{l}\text { Step } 4 \\
\text { SRlca }=6.50-0.092 \mathrm{EF}-0.030 \% \Delta d \text { br. art. }+0.053 \\
\text { DPAP }-0.64 \mathrm{~V}_{\mathrm{E}} \mathrm{N}_{\mathrm{A}} \\
\mathrm{R}=0.63, \mathrm{p}=0.001 ; \text { St. error of estimate }=1.09\end{array}$ \\
\hline $\begin{array}{l}\text { Step } 5 \\
\text { SRlca }=4.25-0.086 \mathrm{EF}-0.030 \% \Delta d \text { br. art. }+0.064 \\
\text { DPAP }-0.55 \mathrm{~V}_{\mathrm{E}} \mathrm{N}_{\mathrm{A}}+0.082 \mathrm{NV} \text { CBP } \mathrm{R}=0.67, \mathrm{p}=0.0009 \\
\text { St. error of estimate }=1.05\end{array}$ \\
\hline
\end{tabular}

Table IX. Analysis of instrumental predictors of the severity of the left CA structural remodelling in patients with $\mathrm{EH}$

\begin{tabular}{|l|c|c|c|}
\hline The factor & $\beta$-coefficient & $\mathbf{R}$ & $\begin{array}{c}\text { Strength of the factor } \\
\text { influence (\%) }\end{array}$ \\
\hline EF & -0.51 & 0.0004 & 31.5 \\
\hline$\% D d$ br. art. & -0.24 & 0.009 & 14.8 \\
\hline DPAP & 0.40 & 0.007 & 25.0 \\
\hline Ve/Na & -0.24 & 0.018 & 14.8 \\
\hline NV CBP & 0.23 & 0.021 & 13.9 \\
\hline
\end{tabular}

groups $[21,25]$. It was found that the magnitude of PBP effected on the appearance of structural changes in the aorta and large vessels, as well as their degree of compliance.

Thus, the remodelling processes of the extracranial vessels were deeply connected with structural and functional changes that occur in the myocardium. However, structural changes in the left and right CA 
did not occur synchronously. Most of the identified factors affected the remodelling of both CA. However, some differences in clinical and instrumental parameters were revealed. So, the remodelling of the left CA was more influenced by high values of DPA and variability of the CBP. The remodelling of the right $\mathrm{CA}$ was influenced by high values of DAP.

\section{Conclusions}

1. Certain asymmetry of extracranial vessels' remodelling was revealed in patients with EH. In patients with uncomplicated $\mathrm{EH}$ most of the changes were defined in the left CA, but not in the right one $(31.8 \%$ versus $15.5 \%, \mathrm{p}<0.05)$. The increase in the degree of hypertension led to the progression of atherosclerotic lesions prevalence mainly in the left CA. The combination of IHD and $\mathrm{EH}$ was associated with an increase of the right CA damage. Changes in the right CA were more extensive in patients with severe $\mathrm{EH}$. They were associated with the severity of the disease rather than depended on the degree of hypertension.

2. The largest structural changes in the left and right CA $(88.2 \%$ and $70.6 \%$, respectively) were determined in patients with a history of a "double catastrophe" - MI and CS. In the presence of $\mathrm{MI}$, atherosclerotic lesions were observed mainly in the left CA $(53.5 \%$ versus $23.9 \%, \mathrm{p}<0.05)$. The appearance of cerebrovascular complications was associated with a significant increase of atherosclerotic lesions not only in the left, but also in the right CA $(50.0 \%$ and $42.9 \%$, respectively; $\mathrm{p}>0.05$ ).

3. Predictors of structural and functional changes in the left and right $\mathrm{CA}$ are the stages of $\mathrm{EH}$ (the strength of influence is $30.3 \%$ and $18.7 \%$, respectively), patients' age (the strength of influence is $55.7 \%$ and $48.0 \%$, respectively) and increased waist circumference (the strength of influence is $14.0 \%$ and $20.3 \%$, respectively). The remodelling of the left CA was more influenced by high values of DPAP, variability of the CBP (the strength of influence is $25.0 \%$ and $13.9 \%$, respectively). The remodelling of the right $\mathrm{CA}$ was influenced by high values of DAP (the strength of the influence is $16.4 \%)$. The remodelling processes of both CA were closely associated with the presence of endothelial dysfunction, structural and functional changes in the myocardium.

Continued research in this direction will allow us to study more deeply the pathogenesis of disorders of structural and functional state of arteries. It will give the possibility to determine the degree of cardiovascular risk in patients with essential hypertension and the presence of carotid arteries remodelling more accurately and to develop preventive and therapeutic measures, which are very important and relevant to modern cardiology.

\section{Conflict of interest: none declared.}

\section{References}

1. Bagmet A.D. Vessel remodeling and apoptosis in the norm and pathology. Kardiologiya 2002; 3: 83-86.

2. Gonchar' A.V., Kovaleva O.N., Khmara A.T. Remodeling of common carotid arteries in patients affected by hypertonic disease and obesity. Nauchniye vedomosti Belgorodskogo gosudarstvennogo universiteta. Seria: Meditsina. Pharmatsia 2013; 23: 73-78.

3. Kuzminova N.V. The remodeling indices of carotid arteries in patients affected by hypertonic disease at various types of myocardium geometry. Visnyk morfologii 2010; 16: 676-682.

4. Kuzminova N.V. Remodeling of extracranial vessels and indices of arterial elasticity in patients affected by hypertonic disease depending on the stage of disease. Visnyk naukovykh doslidzen 2010; 3: 10-13.

5. Polyvoda S.N., Kolesnyk U.M., Cherepok A.A. The affection of target organs at hypertonic disease. Practical guidelines. Chetverta khvylia, Kyev 2005: 800.

6. Park S., Lakatta E.G. Role of inflammation in the pathogenesis of arterial stiffness. Yonsei Med. J. 2012; 53: 258-261.

7. Melnikova L.V. Structural-functional features of carotid arteries in patients affected essential hypertension depending on the value of the systolic arterial pressure. Arterial hypertension 2009; 15: 551-554.

8. Berk B.C. Vascular smooth muscle growth: autocrine growth mechanisms. Physiol. Rev. 2001; 81: 999-1030.

9. Cupini L.M., Pasqualetti P., Diomedi M. et al. Carotid artery intima-media thickness and lacunar versus nonlacunar infarcts. Stroke 2002; 33: 689-694.

10. Nikic P., Savic M., Jakovljevic V., Djuric D. Intima-media thickness of common carotid artery, carotid atherosclerosis and subtypes of ischemic cerebral disease. Rom. J. Intern. Med. 2004; 42: 149-160.

11. Touboul P.J., Hennerici M.G., Meairs S. et al. Mannheim intima-media thickness and plaque consensus (2004-2006-2011). An update on behalf of the Advisory Board of the 3rd, 4th and 5th Watching the Risk Symposia, at the 13th, 15th and 20th European Stroke Conferences, Mannheim, Germany, 2004, Brussels, Belgium, 2006, and Hamburg, Germany, 2011. Cerebrovasc. Dis. 2012; 34: 290-296.

12. Kazmierski R., Watala C., Lukasik M. et al. Common carotid artery remodeling studied by sonomorphological criteria. J. Neuroimaging 2004; 14: 258-264.

13. Mancia G., De Backer G., Dominiczak A. et al. 2007 Guidelines for the Management of Arterial Hypertension: The Task Force for the Management of Arterial Hypertension of the European Society of Hypertension (ESH) and of the European Society of Cardiology (ESC). J. Hypertens. 2007; 26: 1105-1187.

14. Feigenbaum Kh. Echocardiography. Vidar, Moscow 1999: 511.

15. American Society of Echocardiography Committee on Standards. Recommendations for quantification of the left ventricle by two dimensional echocardiography. J. Amer. Soc. Echo. 1989; 2: 358-367.

16. Ganau A., Devereux R.B., Roman M.J. et al. Patterns of left ventricular hypertrophy and geometric remodeling in essential hypertension. J. Am. Coll. Cardiol. 1992; 19: 1550-1558.

17. Kindermann M. How to diagnose diastolic heart failure: a consensus statement on the diagnosis of heart failure with normal left ventricular ejection fraction by the Heart Failure and Echocardiography 
Associations of the European Society of Cardiology. Eur. Heart. J. 2007; 28: 2686.

18. Dziak G.V., Kolesnik T.V., Pogoretskyi U.N. Round-the clock monitoring of the arterial pressure. Dnepropetrovsk 2005: 200.

19. Leliuk V.G., Leliuk S.E. The main principles of hemodynamics and ultrasound vessel examination. In: Mitkova V.V. (ed.). Klinicheskoe rukovodstvo po ultrazvukovoi diagnostike. Vidar, Moscow 1997: 185-220.

20. Kaprio J., Norio R., Personen E. et al. Intimal thickening of the coronary arteries in infants in relation to family history of coronary artery disease. Circulation 1993; 87: 1960-1968.

21. Celermajer D.S., Sorensen K.E., Gooch V.M. et al. Non-invasive detection of endothelial dysfunction in children and adults at risk of atherosclerosis. Lancet 1992; 340: 1111-1115.
22. Rebrova O.U. Statistical analysis of medical data. Applying software kit STATISTICA. MediaSfera, Moscow 2006: 312.

23. Patent 43703 UA, MPK A 61 B 5/00. The way of diagnosing the severity of atherosclerotic affection of carotid arteries and related to it unfavorable prognosis of the disease in hypertonic disease affected patients. Kuzminova Nataliya Vitaliyivna; applicant and patent owner of VNMU named after M.I. Pyrogov. u 200903489; appl. 10.04.09; published. 25.08.09, Bulletin. 16.

24. Kuzminova N.V. The indices of vascular remodeling in patients affected by hypertonic disease depending on the degree of arterial hypertension. Medizina i ... 2009; 23: 11-16.

25. Kuzminova N.V. Morpholgical changes in vascular wall in patients affected by hypertonic disease depending on the type of complications. Visnyk morfologii 2009; 15: 150-154. 